\title{
$\mu$-CONSTANT DEFORMATIONS OF FUNCTIONS ON ICIS
}

\author{
R. S. CARVALHO, B. ORÉFICE-OKAMOTO, J. N. TOMAZELLA
}

\begin{abstract}
We study deformations of holomorphic function germs $f:(X, 0) \rightarrow \mathbb{C}$ where $(X, 0)$ is an ICIS. We present conditions for these deformations to have constant Milnor number, Euler obstruction and Bruce-Roberts number.
\end{abstract}

\section{INTRODUCTION}

Let $f:\left(\mathbb{C}^{n}, 0\right) \rightarrow(\mathbb{C}, 0)$ be a holomorphic function germ with isolated singularity and let $F:\left(\mathbb{C}^{n} \times \mathbb{C}, 0\right) \rightarrow(\mathbb{C}, 0)$ be a deformation of $f$.

We denote, for each $t \in \mathbb{C}, f_{t}:\left(\mathbb{C}^{n}, 0\right) \rightarrow(\mathbb{C}, 0)$ the germ defined by $f_{t}(x)=F(x, t)$.

Then, we have a family of function germs, $f_{t}$. Many authors studied the properties of such a family and a very important result is to know when the family has constant topological type. In this direction, we have the Milnor number ([16]), which is a well known number related to a function germ. We know that a family $f_{t}$ has constant topological type if and only if, it has constant Milnor number ([13], [24]).

However, the problem of determining if a family has constant Milnor number is not easy. Greuel [10, p.161] presents algebraic methods to verify if a family has constant Milnor number in the following theorem.

Theorem 1.1. [10, Theorem 1.1] Let $f:\left(\mathbb{C}^{n}, 0\right) \rightarrow(\mathbb{C}, 0)$ be a holomorphic function germ with isolated singularity at the origin. For any deformation $F:\left(\mathbb{C}^{n} \times \mathbb{C}\right) \rightarrow(\mathbb{C}, 0)$ of $f$ the following statements are equivalent:

(1) $F$ is $\mu$-constant.

(2) For every holomorphic curve $\gamma:(\mathbb{C}, 0) \rightarrow\left(\mathbb{C}^{n} \times \mathbb{C}, 0\right)$

$$
\nu((\partial F / \partial t) \circ \gamma)>\inf \left\{\nu\left(\left(\partial F / \partial x_{i}\right) \circ \gamma\right) \mid i=1, \ldots, n\right\},
$$

(where $\nu$ denotes the usual valuation of a complex curve).

(3) Same statement as in (2) with "> " replaced by " $\geq$ ".

(4) $\partial F / \partial t \in \bar{J}$, (where $\bar{J}$ denotes the integral closure of the Jacobian ideal $J=$ $\left\langle\partial F / \partial x_{1}, \ldots, \partial F / \partial x_{n}\right\rangle$ in $\left.\mathcal{O}_{n+1}\right)$.

(5) $\partial F / \partial t \in \sqrt{J}$, (where $\sqrt{J}$ denotes the radical of $J$ ).

(6) $v(J)=\left\{(x, t) \in \mathbb{C}^{n} \times \mathbb{C} \mid\left(\partial F / \partial x_{i}\right)(x, t)=0, i=1, \ldots, n\right\}=\{0\} \times \mathbb{C}$ near $(0,0)$.

Given an analytic variety germ $(X, 0)$ and a function germ $f:\left(\mathbb{C}^{n}, 0\right) \rightarrow(\mathbb{C}, 0)$ we have the Bruce-Roberts number, $\mu_{B R}(X, f)([3])$. The Bruce-Roberts number generalizes the Milnor number in the sense that the germ is $\mathcal{R}_{X}$-finitely determined if and only if the Bruce-Roberts number is finite, where $\mathcal{R}_{X}$ is the group of the diffeomorphisms which preserves $(X, 0)$.

The first author was partially supported by CAPES. The second author was partially supported by FAPESP Grant 2016/25730-0. The third author was partially supported by CNPq Grant 309086/2017-5 and FAPESP Grant 2016/04740-7. 
In [1], Ahmed, Ruas and Tomazella study the Theorem 1.1 for the Bruce-Roberts number.

In [11], Hamm introduces the Milnor number of an isolated complete intersection singularity (ICIS). The constancy of the topological type implies the constancy of the Milnor number of a family of ICIS of dimension $d \neq 2$ (see [20]).

If $(X, 0) \subset\left(\mathbb{C}^{n}, 0\right)$ is an ICIS and $f:(X, 0) \rightarrow(\mathbb{C}, 0)$ is a holomorphic function germ with isolated singularity, we can, also, look to the Milnor number of $f:(X, 0) \rightarrow(\mathbb{C}, 0)$, $\mu\left(\left.f\right|_{X}\right)$. By the Lê-Greuel formula (see [14, p.77])

$$
\mu\left(\left.f\right|_{X}\right)=\mu(X, 0)+\mu\left(X \cap f^{-1}(0), 0\right),
$$

where $\mu(X, 0)$ and $\mu\left(X \cap f^{-1}(0), 0\right)$ denote the Milnor number of the ICIS defined by Hamm. Therefore, to study the constancy of $\mu\left(X \cap f_{t}^{-1}(0)\right)$ is equivalent to study the constancy of $\mu\left(\left.f_{t}\right|_{X}\right)$.

In this work, we study the constancy of the Milnor number of such a family, $f_{t}$ : $(X, 0) \rightarrow(\mathbb{C}, 0)$. That is, we analyze the assertions of Theorem 1.1 for this singular case.

We apply this study to produce examples of families of function in an ICIS which have constant Milnor number. Moreover, we present conditions of the constancy of the local Euler obstruction and of the Bruce-Roberts number. Furthermore, we present a sufficient condition for family $f_{t}$ to be $C^{0}-\mathcal{R}_{X}$-trivial.

Finally, we analyze the constancy of Milnor number of a family $f_{t}:\left(X_{t}, 0\right) \rightarrow(\mathbb{C}, 0)$ where $\left(X_{t}, 0\right)$ is a deformation of ICIS $(X, 0)$. For this study we use the strict integral closure of a module and we analyze the assertions of Theorem 1.1 which make sense in this new context.

\section{PRELiminary CONCEPTS}

Let $(X, 0) \subset\left(\mathbb{C}^{n}, 0\right)$ be the ICIS defined by the zero set of a holomorphic map germ $\phi:\left(\mathbb{C}^{n}, 0\right) \rightarrow\left(\mathbb{C}^{p}, 0\right)$, with $n>p$.

Let $\Phi:\left(\mathbb{C} \times \mathbb{C}^{n}, 0\right) \rightarrow\left(\mathbb{C}^{p}, 0\right)$ be a holomorphic deformation of $\phi$, defined by $\Phi(s, z)=$ $\phi_{s}(z)$, where $\phi_{0}=\phi$ and $X_{s}:=\phi_{s}^{-1}(0)$ is smooth for $s \neq 0$ small enough. We denote $\mathcal{X}=\Phi^{-1}(0)$. Let $f:(X, 0) \rightarrow(\mathbb{C}, 0)$ be a holomorphic function germ and let $F:(\mathcal{X}, 0) \rightarrow$ $(\mathbb{C}, 0)$ such that for all $s \neq 0$ small enough, the germ

$$
\begin{aligned}
& f_{s}:\left(X_{s}, 0\right) \rightarrow(\mathbb{C}, 0) \\
& z \quad \mapsto F(s, z)
\end{aligned}
$$

is a Morse function. Inspired by [16], we define the Milnor number of $f$ by

$$
\mu\left(\left.f\right|_{X}\right)=\sharp S\left(f_{s}\right),
$$

where $f_{s}:\left(X_{s}, 0\right) \rightarrow(\mathbb{C}, 0)$ is a morsification of $f:(X, 0) \rightarrow(\mathbb{C}, 0)$, with $\left(X_{s}, 0\right)$ a smoothing of $(X, 0)$ and $S\left(f_{s}\right)$ denotes the set of singular points of $f_{s}$.

In order to calculate this number, let $D=\left\{(s, z) \in \mathcal{X} \mid z\right.$ is a singular point of $\left.f_{s}\right\}$ and $\pi: D \rightarrow \mathbb{C}$ the restriction of the projection on the first coordinate. We have that

$$
\mu\left(\left.f\right|_{X}\right)=\sharp S\left(f_{s}\right)=\operatorname{degree}(\pi) .
$$

Therefore, from [17],

$$
\mu\left(\left.f\right|_{X}\right)=e\left(\langle s\rangle, \frac{\mathcal{O}_{\mathcal{X}, 0}}{J\left(f_{s}, \phi_{s}\right)}\right),
$$

where $\mathcal{O}_{\mathcal{X}, 0}$ is the local ring of $(\mathcal{X}, 0), J\left(f_{s}, \phi_{s}\right)$ is the ideal generated by the maximal order minors of the Jacobian matrix of $\left(f_{s}, \phi_{s}\right)$ (partial derivatives with respect to $z$, only) and $e(I, R)$ denotes the Hilbert-Samuel multiplicity of the ideal I with respect to the ring $\mathrm{R}$.

Since $\frac{\mathcal{O}_{\mathcal{X}, 0}}{J\left(f_{s}, \phi_{s}\right)}$ is a determinantal ring, it is Cohen-Macaulay, therefore, from [15, p.138] 
$\mu\left(\left.f\right|_{X}\right)=\operatorname{dim}_{\mathbb{C}} \frac{\mathcal{O}_{\mathcal{X}, 0}}{\langle s\rangle+J\left(f_{s}, \phi_{s}\right)}=\operatorname{dim}_{\mathbb{C}} \frac{\frac{\mathcal{O}_{n+1}}{\langle\Phi\rangle}}{\langle s\rangle+J\left(f_{s}, \phi_{s}\right)}=\operatorname{dim}_{\mathbb{C}} \frac{\mathcal{O}_{n}}{\langle\phi\rangle+J(f, \phi)}=\mu(X, 0)+\mu\left(X \cap f^{-1}(0), 0\right)$, where $\mathcal{O}_{k}$ denotes the local ring of the function germs from $\left(\mathbb{C}^{k}, 0\right)$ to $\mathbb{C}$ and the last equality is the Lê-Greuel formula.

In order to produce a result like Theorem 1.1 for this Milnor number, we use the following result of Teissier, which gives us a characterization for the integral closure of an ideal of the ring $\mathcal{O}_{X, x}$, where $(X, x)$ is an analytic variety.

Theorem 2.1. [23, Proposition 0.4] Let $(X, x) \subset\left(\mathbb{C}^{n}, 0\right)$ be an analytic variety and let I be an ideal in $\mathcal{O}_{X, x}$, the following conditions are equivalent:

(i) $h \in \bar{I}$, where $\bar{I}=\left\{h \in \mathcal{O}_{\mathbb{C} \times X} \mid \exists a_{i} \in I^{i}\right.$ with $\left.h^{k}+a_{1} h^{k-1}+\ldots+a_{k-1} h+a_{k}=0\right\}$.

(ii) For each system of generators $h_{1}, \ldots, h_{r}$ of $I$ there exists a neighborhood $U$ of 0 and a constant $c>0$ such that $|h(x)| \leq \operatorname{csup}\left\{\left|h_{1}(x)\right|, \ldots,\left|h_{r}(x)\right|\right\}, \forall x \in U$.

(iii) (Valuation Criterion) For each analytic curve $\gamma:(\mathbb{C}, 0) \rightarrow(X, x), h \circ \gamma \in$ $\left(\gamma^{*}(I)\right) \mathcal{O}_{1}$, where $\left(\gamma^{*}(I)\right) \mathcal{O}_{1}$ is the ideal generated by $h_{i} \circ \gamma, i=1, \ldots, r$.

(iv) $\nu(h \circ \gamma) \geq \inf \left\{\nu\left(h_{1} \circ \gamma\right), \ldots, \nu\left(h_{r} \circ \gamma\right)\right\}$, for $\nu$ being the usual valuation of a complex curve.

\section{MAin RESUlts}

Let $(X, 0) \subset\left(\mathbb{C}^{n}, 0\right)$ be the ICIS defined by a holomorphic map germ $\phi:\left(\mathbb{C}^{n}, 0\right) \rightarrow$ $\left(\mathbb{C}^{p}, 0\right)$ and let $f:(X, 0) \rightarrow(\mathbb{C}, 0)$ be a holomorphic function germ with isolated singularity. Consider

$$
\begin{aligned}
F:(\mathbb{C} \times X, 0) & \rightarrow(\mathbb{C}, 0) \\
(t, x) & \mapsto f_{t}(x)
\end{aligned}
$$

a (flat) deformation of $f$. We say that $F$ is $\mu$-constant if $\mu\left(\left.f_{t}\right|_{X}\right)=\mu\left(\left.f\right|_{X}\right)$ for $t$ small enough.

The main goal of this work is to study when $F$ is $\mu$-constant, that is, to present a result like Theorem 1.1 for a family of functions on an ICIS.

The assertions of the Theorem 1.1 in this context would be

$\left(1_{X}\right) F$ is $\mu$-constant.

$\left(2_{X}\right)$ For every holomorphic curve $\gamma:(\mathbb{C}, 0) \rightarrow(\mathbb{C} \times X, 0)$

$$
\nu((\partial F / \partial t) \circ \gamma)>\inf \left\{\nu\left(B_{i} \circ \gamma\right) \mid i=1, \ldots, r\right\} \text { in } \mathcal{O}_{\mathbb{C} \times X},
$$

(where $\nu$ denotes the usual valuation of a complex curve).

$\left(3_{X}\right)$ Same statement as in $(2)$ with " $>$ " replaced by " $\geq$ ".

$\left(4_{X}\right) \partial F / \partial t \in \overline{J_{X}}$ in $\mathcal{O}_{\mathbb{C} \times X}$.

$\left(5_{X}\right) \partial F / \partial t \in \sqrt{J_{X}}$ in $\mathcal{O}_{\mathbb{C} \times X}$.

$\left(6_{X}\right) v\left(J_{X}\right)=\left\{(t, x) \in \mathbb{C} \times \mathbb{C}^{n} \mid B_{i}(t, x)=0, i=1, \ldots, r\right\}=\mathbb{C} \times\{0\}$ near $(0,0)$, where $B_{1}, \ldots, B_{r}$ are the maximal order minors of the Jacobian matrix of $\left(f_{t}, \phi\right)$ (partial derivatives with respect to $x$, only) and $J_{X}$ is the ideal generated by them.

Unfortunately, in this singular context, these assertions are not equivalent. But in this section we show

$$
\begin{aligned}
\left(2_{X}\right) \Rightarrow & \left(3_{X}\right) \Leftrightarrow\left(4_{X}\right) \Rightarrow\left(5_{X}\right), \\
\left(1_{X}\right) \Leftrightarrow\left(6_{X}\right) & \left(6_{X}\right) \Rightarrow\left(5_{X}\right) \\
\left(4_{X}\right) & \Rightarrow\left(1_{X}\right) .
\end{aligned}
$$

Also, we present examples for 


$$
\begin{aligned}
&\left(1_{X}\right) \nRightarrow\left(2_{X}\right), \\
&\left(1_{X}\right) \nRightarrow\left(3_{X}\right), \\
&\left(3_{X}\right) \nRightarrow\left(2_{X}\right), \\
&\left(5_{X}\right) \nRightarrow\left(2_{X}\right), \\
&\left(5_{X}\right) \nRightarrow\left(1_{X}\right), \\
&\left(5_{X}\right) \nRightarrow\left(3_{X}\right) .
\end{aligned}
$$

Although the integral closure that appears in the assertion $\left(4_{X}\right)$ is the one of an ideal we need to work with integral closure of submodule to show that $\left(4_{X}\right)$ implies $\left(1_{X}\right)$. Therefore, we remember, now, its definition.

Definition 3.1. [5, Definition 1.3] Suppose $(X, 0)$ is a complex analytic germ, $M$ a submodule of $\mathcal{O}_{X, 0}^{p}$. Then $h \in \mathcal{O}_{X, 0}^{p}$ is in the integral closure of $M$, denoted by $\bar{M}$, if and only if for all $\gamma:(\mathbb{C}, 0) \rightarrow(X, 0), h \circ \gamma \in\left(\gamma^{*}(M)\right) \mathcal{O}_{1}$.

Replacing $\mathcal{O}_{1}$ by its maximal ideal $\mathcal{M}_{1}$ we get the definition of strict integral closure of $M$, which is denoted by $\bar{M}^{\dagger}$ (see [4, Definition 1.1]). In this case, $h \in \bar{M}^{\dagger}$ it is said strictly dependent on $M$.

Our next result provides a way to ensure the constancy of $\mu\left(X \cap f_{t}^{-1}(0), 0\right)$.

Theorem 3.2. Let $(X, 0) \subset\left(\mathbb{C}^{n}, 0\right)$ be an ICIS defined by $\phi:\left(\mathbb{C}^{n}, 0\right) \rightarrow\left(\mathbb{C}^{p}, 0\right)$ and let $f:(X, 0) \rightarrow(\mathbb{C}, 0)$ be a holomorphic germ with isolated singularity. Consider $F$ : $(\mathbb{C} \times X, 0) \rightarrow(\mathbb{C}, 0)$ a deformation of $f$ and $G: \mathbb{C} \times \mathbb{C}^{n} \rightarrow \mathbb{C} \times \mathbb{C}^{p}$ given by $G(t, x)=$ $(F(t, x), \phi(x))$. If $\partial F / \partial t \in \overline{J_{X}}$ in $\mathcal{O}_{\mathbb{C} \times X}$ then:

(i) $(\partial F / \partial t, 0) \in \overline{\left\{x_{i}\left(\partial G / \partial x_{j}\right)\right\}}$ in $\mathcal{O}_{X \cap f_{t}^{-1}(0)}$ with $i, j=1, \ldots, n$, where $\overline{\left\{x_{i}\left(\partial G / \partial x_{j}\right)\right\}}$ denotes the integral closure of the $\mathcal{O}_{X}$-module $\left\{x_{i}\left(\partial G / \partial x_{j}\right)\right\}$.

(ii) $X \cap f_{t}^{-1}(0)$ is an ICIS with $\mu\left(X \cap f_{t}^{-1}(0), 0\right)$ constant.

Proof: (i) We remember that $J_{X}$ is the ideal in $\mathcal{O}_{\mathbb{C} \times X}$ generated by the minors of order $p+1$ of the matrix

$$
\left(\begin{array}{cccc}
f_{t_{x_{1}}} & f_{t_{x_{2}}} & \cdots & f_{t_{x_{n}}} \\
\phi_{1_{x_{1}}} & \phi_{1_{x_{2}}} & \cdots & \phi_{1_{x_{n}}} \\
\vdots & \vdots & \vdots & \vdots \\
\phi_{p_{x_{1}}} & \phi_{p_{x_{2}}} & \cdots & \phi_{p_{x_{n}}}
\end{array}\right) \text {, where } \phi=\left(\phi_{1}, \ldots, \phi_{p}\right) \text { and } f_{t}(x)=F(t, x)
$$

These minors are

$$
\begin{aligned}
& B_{v}=\left|\begin{array}{cccc}
f_{t_{x_{1}}} & f_{t_{x_{2}}} & \cdots & f_{t_{x_{j_{p+1}}}} \\
\phi_{1 x_{j_{1}}} & \phi_{1 x_{j_{2}}} & \cdots & \phi_{1 x_{j_{p+1}}} \\
\vdots & \vdots & \vdots & \vdots \\
\phi_{p_{x_{j_{1}}}} & \phi_{p_{x_{j_{2}}}} & \cdots & \phi_{p_{x_{j_{p+1}}}}
\end{array}\right|=f_{t_{x_{j_{1}}}}\left|\begin{array}{cccc}
\phi_{1 x_{j_{2}}} & \phi_{1_{x_{j_{3}}}} & \cdots & \phi_{1 x_{j_{p+1}}} \\
\vdots & \vdots & \vdots & \vdots \\
\phi_{p_{x_{j_{2}}}} & \phi_{p_{x_{j_{3}}}} & \cdots & \phi_{p_{x_{j_{p+1}}}}
\end{array}\right|- \\
& f_{t_{x_{j_{2}}}}\left|\begin{array}{cccc}
\phi_{1_{x_{1}}} & \phi_{1_{x_{j_{3}}}} & \cdots & \phi_{1_{x_{j_{p}}}} \\
\vdots & \vdots & \vdots & \vdots \\
\phi_{p_{x_{j_{1}}}} & \phi_{p_{x_{j_{3}}}} & \cdots & \phi_{p_{x_{j_{p}}}}
\end{array}\right|+\ldots+(-1)^{p+2} f_{t_{x_{j_{p+1}}}}\left|\begin{array}{cccc}
\phi_{1_{x_{1}}} & \phi_{1_{x_{j_{2}}}} & \cdots & \phi_{1_{x_{j_{p}}}} \\
\vdots & \vdots & \vdots & \vdots \\
\phi_{p_{x_{j_{1}}}} & \phi_{p_{x_{j_{2}}}} & \cdots & \phi_{p_{x_{j_{p}}}}
\end{array}\right|,
\end{aligned}
$$

for each vector $v=\left(j_{1}, \ldots, j_{p+1}\right)$ with $j_{1}<\ldots<j_{p+1}$ and $j_{1}, \ldots, j_{p+1} \in\{1, \ldots, n\}$.

$$
\text { Let } A_{v_{i}}=\left|\begin{array}{cccccc}
\phi_{1_{x_{1}}} & \cdots & \phi_{1_{x_{j_{i-1}}}} & \phi_{1_{x_{j_{i+1}}}} & \cdots & \phi_{1_{x_{j_{p+1}}}} \\
\vdots & \vdots & \vdots & \vdots & \vdots & \vdots \\
\phi_{p_{x_{j_{1}}}} & \cdots & \phi_{p_{x_{j_{i-1}}}} & \phi_{p_{x_{j_{i+1}}}} & \cdots & \phi_{p_{x_{j_{p+1}}}}
\end{array}\right| \text {, for each } i=1, \ldots, p+1 \text {. }
$$


Thus $B_{v}=f_{t_{x_{j_{1}}}} A_{v_{1}}-f_{t_{x_{j_{2}}}} A_{v_{2}}+\ldots+(-1)^{1+p+1} f_{t x_{j_{p+1}}} A_{v_{p+1}}$. Then $\left(\sum_{i=1}^{p+1}(-1)^{1+i} f_{t_{x_{j}}} A_{v_{i}}, \sum_{i=1}^{p+1}(-1)^{1+i} \phi_{1_{x_{j_{i}}}} A_{v_{i}}, \sum_{i=1}^{p+1}(-1)^{1+i} \phi_{2_{x_{j_{i}}}} A_{v_{i}}, \ldots, \sum_{i=1}^{p+1}(-1)^{1+i} \phi_{p_{x_{j_{i}}}} A_{v_{i}}\right)=$ $\left(B_{v}, 0, \ldots, 0\right)$, because all the entries, but the first, is the determinant of a matrix with two equal lines.

Thus, $\left(B_{v}, 0, \ldots, 0\right) \in \mathcal{M}_{n} L_{i}$ for $i=1, \ldots, n$, where $L_{i}:=\partial G / \partial x_{i}=\left(f_{t_{x_{i}}}, \phi_{1 x_{i}}, \ldots, \phi_{p_{x_{i}}}\right)$ and $\mathcal{M}_{n}=\left\{h \in \mathcal{O}_{n} \mid h(0)=0\right\}$.

By the hypothesis, $\partial F / \partial t \in \overline{J_{X}}$ in $\mathcal{O}_{\mathbb{C} \times X}$ then, by Theorem 2.1 , for all $\varphi:(\mathbb{C}, 0) \rightarrow \mathbb{C} \times$ $X,(\partial F / \partial t) \circ \varphi \in\left\langle B_{v} \circ \varphi\right\rangle$ and, therefore, $((\partial F / \partial t) \circ \varphi, 0) \in\left\langle\left(B_{v} \circ \varphi, 0\right)\right\rangle \subset\left(\mathcal{M}_{n} L_{i}\right) \circ \varphi$, for $v=\left(j_{1}, \ldots, j_{p+1}\right)$ with $j_{1}<\ldots<j_{p+1}$ and $j_{1}, \ldots, j_{p+1} \in\{1, \ldots, n\}$. Thus, $(\partial F / \partial t, 0) \in$ $\overline{\left\{x_{i}\left(\partial G / \partial x_{j}\right)\right\}}$.

(ii) By the item (i), $(\partial F / \partial t, 0) \in \overline{\left\{x_{i}\left(\partial G / \partial x_{j}\right)\right\}}$ in $\mathcal{O}_{X \cap f_{t}^{-1}(0)}$. Then, $\left\{X \cap f_{t}^{-1}(0)\right\}$ is Whitney regular (see [5, Theorem 2.5]). In particular, $\mu\left(X \cap f_{t}^{-1}(0), 0\right)$ is constant (see [6, Theorem 6.1]).

From the proof of the item (ii) of the previous theorem, $(\partial F / \partial t, 0) \in \overline{\left\{x_{i}\left(\partial G / \partial x_{j}\right)\right\}}$ implies that $\mu\left(X \cap f_{t}^{-1}(0), 0\right)$ is constant.

We see, in the following example that $(\partial F / \partial t, 0) \in \overline{\left\{\partial G / \partial x_{j}\right\}}$ does not imply that $F$ is $\mu$-constant

Example 3.3. Let $(X, 0) \subset\left(\mathbb{C}^{3}, 0\right)$ be the zero set of $\phi(x, y, z)=x^{5}+y^{3}+z^{2}$ and let $f:(X, 0) \rightarrow(\mathbb{C}, 0)$ be defined by $f(x, y, z)=x y$. Consider the deformation of $f$, $F(t,(x, y, z))=x y-t z$. Note that $J_{X}=\left\langle-2 x z-3 t y^{2},-2 y z-5 t x^{4}, 3 y^{3}-5 x^{5}\right\rangle$.

Now let's consider $\gamma:(\mathbb{C}, 0) \rightarrow(\mathbb{C} \times X, 0)$ given by $\gamma(s)=\left(0,-s^{2}, 0, s^{5}\right)$. We have that $\nu((\partial F / \partial t) \circ \gamma)=5$ and $\nu\left(J_{X} \circ \gamma\right)=7$.

Therefore, $\nu((\partial F / \partial t) \circ \gamma)<\nu\left(J_{X} \circ \gamma\right)$. Thus, $\partial F / \partial t \notin \overline{J_{X}}$.

On the other hand, we have that

$(\partial F / \partial t, 0)=(-z, 0)$ and $\{\partial G / \partial x, \partial G / \partial y, \partial G / \partial z\}=\left\{\left(y, 5 x^{4}\right),\left(x, 3 y^{2}\right),(-t, 2 z)\right\}$, where $G(t,(x, y, z))=(F(t,(x, y, z)), \phi(x, y, z))$.

Note that

$$
\begin{gathered}
(-z, 0) \in \overline{\{\partial G / \partial x, \partial G / \partial y, \partial G / \partial z\}} \text { if and only if } \\
J_{2}((-z, 0),\{\partial G / \partial x, \partial G / \partial y, \partial G / \partial z\}) \subset \overline{J_{2}(\{\partial G / \partial x, \partial G / \partial y, \partial G / \partial z\})}
\end{gathered}
$$

(see [5, Proposition 1.7]), where $J_{2}((-z, 0),\{\partial G / \partial x, \partial G / \partial y, \partial G / \partial z\})$ are the minors $2 \times 2$ of matrix

$$
\left(\begin{array}{cccc}
-z & y & x & -t \\
0 & 5 x^{4} & 3 y^{2} & 2 z
\end{array}\right)
$$

Therefore, we just show that

$$
-5 x^{4} z,-3 y^{2} z \text { and }-2 z^{2} \text { belong to } \overline{\left\langle-2 x z-3 t y^{2},-2 y z-5 t x^{4}, 3 y^{3}-5 x^{5}\right\rangle} .
$$

Let $\gamma:(\mathbb{C}, 0) \rightarrow(\mathbb{C} \times X, 0), \gamma(s)=\left(\gamma_{1}(s), \gamma_{2}(s), \gamma_{3}(s), \gamma_{4}(s)\right)$. Thus, $\gamma_{2}^{5}+\gamma_{3}^{3}+\gamma_{4}^{2}=0$.

By the Theorem 2.1 we have the desired.

Therefore, $(\partial F / \partial t, 0) \in \overline{\{\partial G / \partial x, \partial G / \partial y, \partial G / \partial z\}}$.

However, $\mu\left(\left.f_{0}\right|_{X}\right)=17$ and $\mu\left(\left.f_{t}\right|_{X}\right)=16$, for $t \neq 0$.

We are now ready to prove our main result. 
Theorem 3.4. Let $(X, 0) \subset\left(\mathbb{C}^{n}, 0\right)$ be an ICIS defined by $\phi:\left(\mathbb{C}^{n}, 0\right) \rightarrow\left(\mathbb{C}^{p}, 0\right)$, let $f:(X, 0) \rightarrow(\mathbb{C}, 0)$ be a germ with isolated singularity and let $f_{t}:(X, 0) \rightarrow(\mathbb{C}, 0)$ be a (flat) deformation of $f$. Then:

$$
\begin{aligned}
\left(2_{X}\right) \Rightarrow\left(3_{X}\right) \Leftrightarrow\left(4_{X}\right) \Rightarrow\left(5_{X}\right) \\
\left(1_{X}\right) \Leftrightarrow\left(6_{X}\right) \\
\left(6_{X}\right) \Rightarrow\left(5_{X}\right) \\
\left(4_{X}\right) \Rightarrow\left(1_{X}\right) .
\end{aligned}
$$

Proof: $\left(2_{X}\right) \Rightarrow\left(3_{X}\right)$ is trivial, $\left(3_{X}\right) \Leftrightarrow\left(4_{X}\right)$ follows from $(i) \Leftrightarrow(i v)$ in Theorem 2.1 and $\left(4_{X}\right) \Rightarrow\left(5_{X}\right)$ because $\overline{J_{X}} \subset \sqrt{J_{X}}$.

$\left(1_{X}\right) \Rightarrow\left(6_{X}\right)$ :

Since $\frac{\mathcal{O}_{n}}{\langle\phi\rangle+J_{X}}$ is Cohen-Macaulay, by the principle of conservation of number and by the hypothesis, we have that

$$
\mu\left(\left.f_{t}\right|_{X}, 0\right)=\mu\left(\left.f\right|_{X}, 0\right)=\sum_{(t, x) \in\{t\} \times v\left(J_{X}\right)} \mu\left(\left.f_{t}\right|_{X}, x\right),
$$

then $\mu\left(\left.f_{t}\right|_{X}, x\right)=0$ for all $x \neq 0$ and therefore $v\left(J_{X}\right)=\mathbb{C} \times\{0\}$ near $(0,0)$.

$\left(6_{X}\right) \Rightarrow\left(1_{X}\right)$ :

By the hypothesis $v\left(J_{X}\right)=\mathbb{C} \times\{0\}$ near $(0,0)$. Again, by the principle of conservation of number, $\mu\left(\left.f\right|_{X}, 0\right)=\mu\left(\left.f_{t}\right|_{X}, 0\right)$. Therefore, $F$ is $\mu$-constant.

$\left(6_{X}\right) \Rightarrow\left(5_{X}\right)$ :

By the hypothesis $v\left(J_{X}\right)=\mathbb{C} \times\{0\}$ near $(0,0)$. We have that $F \in \mathcal{M}_{n}$, then $\left.(\partial F / \partial t)\right|_{v\left(J_{X}\right)}=\left.(\partial F / \partial t)\right|_{\mathbb{C} \times\{0\}} \equiv 0$. Thus, $v\left(J_{X}\right) \subset v(\partial F / \partial t)$. Therefore, $\partial F / \partial t \in \sqrt{J_{X}}$ by the Hilbert Nullstellensatz Theorem.

$\left(4_{X}\right) \Rightarrow\left(1_{X}\right)$ :

By the Lê-Greuel formula, $\mu\left(\left.f_{t}\right|_{X}\right)=\mu(X, 0)+\mu\left(X \cap f_{t}^{-1}(0), 0\right)$. Therefore, since, $\mu\left(X \cap f_{t}^{-1}(0), 0\right)$ is constant by the Theorem [3.2, $\mu\left(\left.f_{t}\right|_{X}\right)$ is also constant.

From here to the end of this section, our goal is to present counterexamples for the other implications.

A good idea to look for such counterexamples is to see families which we know, at first, that have constant Milnor number. For this, we recall the results of [18] on deformations of weighted homogeneous germs.

We say that $f=\left(f_{1}, \ldots, f_{p}\right):\left(\mathbb{C}^{n}, 0\right) \rightarrow\left(\mathbb{C}^{p}, 0\right)$ is weighted homogeneous of type $\left(w_{1}, \ldots, w_{n} ; d_{1}, \ldots, d_{p}\right)$ if given $\left(w_{1}, \ldots, w_{n} ; d_{1}, \ldots, d_{p}\right)$ with $w_{i}, d_{j} \in \mathbb{Q}^{+}$we have that for all $\lambda \in \mathbb{C}-\{0\}:$

$$
f\left(\lambda^{w_{1}} x_{1}, \ldots, \lambda^{w_{n}} x_{n}\right)=\left(\lambda^{d_{1}} f_{1}(x), \ldots, \lambda^{d_{p}} f_{p}(x)\right) .
$$

We call $d_{j}$ the weighted degree of $f_{j}$, which is denoted by $w t\left(f_{j}\right)$ and $w_{i}$ is called weight of the variable $x_{i}$.

Let $(X, 0) \subset\left(\mathbb{C}^{n}, 0\right)$ be a germ of analytic variety defined by $\phi:\left(\mathbb{C}^{n}, 0\right) \rightarrow\left(\mathbb{C}^{p}, 0\right)$. If $\phi$ is weighted homogeneous we say $(X, 0)$ is weighted homogeneous.

A non-zero polynomial germ $f:\left(\mathbb{C}^{n}, 0\right) \rightarrow \mathbb{C}$ can be written

$$
f=f_{d}+f_{d+1}+\ldots+f_{l},
$$

where $f_{d} \neq 0$ and each $f_{i}$ is weighted homogeneous of degree $i$. We say that $f_{d}$ is the initial part of $f$, which is denoted by $i n(f)$. 
Moreover, if $f:\left(\mathbb{C}^{n}, 0\right) \rightarrow(\mathbb{C}, 0)$ is a polynomial function and $f_{t}:\left(\mathbb{C}^{n}, 0\right) \rightarrow(\mathbb{C}, 0)$ a deformation of $f$ we have that $f_{t}$ can be written as:

$$
f_{t}(x)=f(x)+\sum_{i=1}^{k} \sigma_{i}(t) \alpha_{i}(x),
$$

with $\alpha_{i}:\left(\mathbb{C}^{n}, 0\right) \rightarrow(\mathbb{C}, 0)$ and $\sigma_{i}:(\mathbb{C}, 0) \rightarrow(\mathbb{C}, 0)$.

If $w t\left(i n\left(\alpha_{i}\right)\right) \geq w t(i n(f))$ for $i=1, \ldots, k$ we say that $f_{t}$ is a non-negative deformation of $f$.

Theorem 3.5. [18, Theorem 4.4] Let $(X, 0) \subset\left(\mathbb{C}^{n}, 0\right)$ be a weighted homogeneous ICIS and let $f:\left(\mathbb{C}^{n}, 0\right) \rightarrow \mathbb{C}$ be a weighted homogeneous germ with an isolated singularity with the same weights of $(X, 0)$ and let $f_{t}$ be a deformation of $f$. If $f_{t}$ is non-negative, then $\mu\left(\left.f_{t}\right|_{X}\right)$ is constant.

Using this result we show in the following example that $\left(1_{X}\right)$ does not imply $\left(2_{X}\right)$ and $\left(1_{X}\right)$ does not imply $\left(3_{X}\right)$.

Example 3.6. Let $(X, 0) \subset\left(\mathbb{C}^{2}, 0\right)$ be defined by $\phi(x, y)=x^{p}-y^{q}$, with $q \geq 3$ and $p>q$, and let $f:(X, 0) \rightarrow(\mathbb{C}, 0)$ be defined by $f(x, y)=x$. Consider the deformation of $f$ defined by $F(t,(x, y))=x+t y$. Note that $J_{X}=\left\langle-q y^{q-1}-p t x^{p-1}\right\rangle$.

Now let's consider $\gamma:(\mathbb{C}, 0) \rightarrow(\mathbb{C} \times X, 0)$ given by $\gamma(s)=\left(0, s^{q}, s^{p}\right)$ and we have that $\nu((\partial F / \partial t) \circ \gamma)=p$ and $\nu\left(J_{X} \circ \gamma\right)=(q-1) p$.

Therefore, $\nu((\partial F / \partial t) \circ \gamma)<\nu\left(J_{X} \circ \gamma\right)$. Thus, $\left(2_{X}\right)$ and $\left(3_{X}\right)$ are not true.

On the other hand since $f_{t}$ is a non-negative deformation, $\mu\left(\left.f_{t}\right|_{X}\right)$ is constant by the Theorem 3.5. Thus, $\left(1_{X}\right)$ is true. 2 ( $)$.

We show in the next example that $\left(3_{X}\right)$ does not imply $\left(2_{X}\right)$ and $\left(5_{X}\right)$ does not imply

Example 3.7. Let $(X, 0) \subset\left(\mathbb{C}^{2}, 0\right)$ be defined by $\phi(x, y)=x^{2 q}-y^{q}$ with $q \geq 2$ and let $f:(X, 0) \rightarrow(\mathbb{C}, 0)$ be defined by $f(x, y)=x^{2 q}+y^{q}$. Consider the deformation of $f$ given by $F(t,(x, y))=x^{2 q}+y^{q}+t x^{4 q-3}$. Note that $J_{X}=\left\langle-4 q^{2} x^{2 q-1} y^{q-1}-q(4 q-3) t x^{4 q-4} y^{q-1}\right\rangle$.

Let $\gamma:(\mathbb{C}, 0) \rightarrow(\mathbb{C} \times X, 0), \gamma(s)=\left(\gamma_{1}(s), \gamma_{2}(s), \gamma_{3}(s)\right)$ such that $\gamma_{2}^{2 q}-\gamma_{3}^{q}=0$. Thus, $\gamma_{2}^{2 q}=\gamma_{3}^{q}$. Then, $2 \nu\left(\gamma_{2}\right)=\nu\left(\gamma_{3}\right)$.

Since $\partial F / \partial t=x^{4 q-3}$, we have that $\nu((\partial F / \partial t) \circ \gamma)=(4 q-3) \nu\left(\gamma_{2}\right)$. Furthermore $\nu\left(J_{X} \circ \gamma\right)=\nu\left(-4 q^{2} \gamma_{2}^{2 q-1} \gamma_{3}^{q-1}-q(4 q-3) \gamma_{1} \gamma_{2}^{4 q-4} \gamma_{3}^{q-1}\right)=(2 q-1) \nu\left(\gamma_{2}\right)+2(q-1) \nu\left(\gamma_{2}\right)=$ $(4 q-3) \nu\left(\gamma_{2}\right)$.

Thus, $\left(3_{X}\right)$ is true, consequently $\left(4_{X}\right)$ is true and $\left(5_{X}\right)$ is true.

Now, let's consider $\gamma:(\mathbb{C}, 0) \rightarrow(\mathbb{C} \times X, 0)$ given by $\gamma(s)=\left(0, s, s^{2}\right)$ and we have that $\nu((\partial F / \partial t) \circ \gamma)=4 q-3$ and $\nu\left(J_{X} \circ \gamma\right)=4 q-3$. Thus, $\left(2_{X}\right)$ is not true. $\left(3_{X}\right)$.

The following example shows that $\left(5_{X}\right)$ does not imply $\left(1_{X}\right)$ and $\left(5_{X}\right)$ does not imply

Example 3.8. Let $(X, 0) \subset\left(\mathbb{C}^{2}, 0\right)$ be defined by $\phi(x, y)=x^{p}-y^{q}$, with $q \geq 3, p<q$ and let $f:(X, 0) \rightarrow(\mathbb{C}, 0)$ be defined by $f(x, y)=x$. Consider the deformation of $f$ given by $F(t,(x, y))=x+t y$. Note that $J_{X}=\left\langle-q y^{q-1}-p t x^{p-1}\right\rangle$.

We have that $\mu\left(\left.f\right|_{X}\right)=p q-p$ and $\mu\left(\left.f_{t}\right|_{X}\right)=p q-q$. Therefore, $\left(1_{X}\right)$ is not true.

Now, let's consider $\gamma:(\mathbb{C}, 0) \rightarrow(\mathbb{C} \times X, 0)$ given by $\gamma(s)=\left(0, s^{q}, s^{p}\right)$. Thus $\nu((\partial F / \partial t) \circ \gamma)=$ $p$ and $\nu\left(J_{X} \circ \gamma\right)=(q-1) p$. Therefore, $\nu((\partial F / \partial t) \circ \gamma)<\nu\left(J_{X} \circ \gamma\right)$.

On the other hand, $\partial F / \partial t \in \sqrt{J_{X}}$ in $\mathcal{O}_{\mathbb{C} \times X}$. 


\section{Some EXAmples}

In this section, we apply our results to produce examples of families of functions in an ICIS which have constant Milnor number although they do not satisfy the hypotheses of the Theorem 3.5. For this we resort to the results about of the Newton polyhedron (see 22 ).

Let $g \in \mathcal{O}_{n}$ then $g$ can be written as $g(x)=\sum a_{k} x^{k}$. We define the support of $g$ as supp $g:=\left\{k \in \mathbb{Z}^{n} \mid a_{k} \neq 0\right\}$ and for $I$ an ideal in $\mathcal{O}_{n}$, we define supp $I:=\bigcup\{\operatorname{supp} g \mid g \in I\}$.

The convex hull in $\mathbb{R}_{+}^{n}$ of the set $\bigcup\left\{k+v \mid k \in \operatorname{supp} I, v \in \mathbb{R}_{+}^{n}\right\}$ is called Newton polyhedron of $I$ and denoted by $\Gamma_{+}(I)$. The union of all compact faces of $\Gamma_{+}(I)$ we denote by $\Gamma(I)$.

Let $\Delta \subset \Gamma_{+}(I)$ be a finite subset, we define $g_{\Delta}=\sum_{k \in \Delta} a_{k} x^{k}$ for any germ $g(x)=$ $\sum a_{k} x^{k}$.

Given $\Delta$ a face of $\Gamma_{+}(I)$, we denote $C(\Delta)$ the cone of half-rays emanating from 0 and passing through $\Delta$. We define $C[[\Delta]]$, the ring of power series with non-zero monomials $x^{k}=x_{1}^{k_{1}} x_{2}^{k_{2}} \cdot \ldots \cdot x_{n}^{k_{n}}$ such that $k=\left(k_{1}, \ldots, k_{n}\right) \in C(\Delta)$. When the ideal generated by $g_{1 \Delta}, g_{2 \Delta}, \ldots, g_{n_{\Delta}}$ has finite codimension in $C[[\Delta]]$ we say that the compact face $\Delta \subset \Gamma(I)$ is Newton non-degenerate. Furthermore, if all compact faces of $\Gamma(I)$ are Newton nondegenerate then the ideal $I$ is said Newton non-degenerate.

Equivalently, in [22, p.2]: $I$ is Newton non-degenerate if for each compact face $\Delta \subset \Gamma(I)$, the equations $g_{1 \Delta}(x)=g_{2 \Delta}(x)=\ldots=g_{n_{\Delta}}(x)=0$ have no common solution in $(\mathbb{C}-\{0\})^{n}$.

Theorem 4.1. [22, Theorem 3.4] Let $I=\left\langle g_{1}, g_{2}, \ldots, g_{s}\right\rangle$ be an ideal of finite codimension in $\mathcal{O}_{n}$. Then $I$ is Newton non-degenerate if and only if $\Gamma_{+}(I)=C(\bar{I})$, where $C(\bar{I})$ is the convex hull in $\mathbb{R}_{+}^{n}$ of the set $\bigcup\left\{m \mid x^{m} \in \bar{I}\right\}$.

Let $(X, 0) \subset\left(\mathbb{C}^{n}, 0\right)$ be the ICIS defined by the zero set of a map germ $\phi:\left(\mathbb{C}^{n}, 0\right) \rightarrow$ $\left(\mathbb{C}^{p}, 0\right)$. Let $f:(X, 0) \rightarrow(\mathbb{C}, 0)$ be a holomorphic function germ with isolated singularity and $F:(\mathbb{C} \times X, 0) \rightarrow(\mathbb{C}, 0)$ a deformation of $f$ given by $F(t, x)=f(x)+t g(x)$, where $g$ is a holomorphic function germ such that $g(0)=0$.

We denote by $B_{1}, \ldots, B_{r}$ the minors of order $p+1$ of $J\left(f_{t}, \phi\right), B_{1}^{0}, \ldots, B_{r}^{0}$ the minors of order $p+1$ of $J(f, \phi)$ and $C_{1}, \ldots, C_{r}$ the minors of order $p+1$ of $J(g, \phi)$. Then, $B_{i}=B_{i}^{0}+t C_{i}$, by the multilinearity of the determinant, for $i=1, \ldots, r$.

With the above notation,

Lemma 4.2. If $g \in \overline{\langle\phi\rangle+J(f, \phi)}$ in $\mathcal{O}_{n+1}$ and $C_{i} \in \overline{\langle\phi\rangle+J(f, \phi)}$ in $\mathcal{O}_{n+1}$ for $i=1, \ldots, r$ then $g \in \overline{J_{X}}$ in $\mathcal{O}_{\mathbb{C} \times X}$.

Proof: Since $C_{i} \in \overline{\langle\phi\rangle+J(f, \phi)}$ then, by Theorem 2.1, there exists a neighborhood $U$ of 0 and a constant $c>0$ such that

$$
|t|\left|C_{i}\right| \leq|t| \operatorname{csup}_{i}\left\{|\phi|,\left|B_{i}^{0}\right|\right\} .
$$

Besides that,

$$
\begin{gathered}
\sup _{i}\left\{|\phi|,\left|B_{i}\right|\right\}=\sup _{i}\left\{|\phi|,\left|B_{i}^{0}+t C_{i}\right|\right\} \geq \\
\sup _{i}\left\{|\phi|,\left|B_{i}^{0}\right|\right\}-|t| \sup _{i}\left\{|\phi|,\left|C_{i}\right|\right\} \geq \\
\sup _{i}\left\{|\phi|,\left|B_{i}^{0}\right|\right\}-|t| \sup _{i}\left\{|\phi|,\left|B_{i}^{0}\right|\right\} \geq \\
(1-\alpha) \sup _{i}\left\{|\phi|,\left|B_{i}^{0}\right|\right\},
\end{gathered}
$$

where $0<\alpha<1$ and $|t| \leq \frac{\alpha}{c}$. 
Thus, $\sup _{i}\left\{|\phi|,\left|B_{i}\right|\right\} \geq K \sup _{i}\left\{|\phi|,\left|B_{i}^{0}\right|\right\}$ for $K>0$ and $t$ small enough. Therefore, $\overline{\langle\phi\rangle+J(f, \phi)} \subseteq \overline{\langle\phi\rangle+J_{X}}$ in $\mathcal{O}_{n+1}$. Then $g \in \overline{\langle\phi\rangle+J_{X}}$ in $\mathcal{O}_{n+1}$. Thus, by Theorem 2.1 for all $\gamma: \mathbb{C} \rightarrow \mathbb{C} \times \mathbb{C}^{n}$ we have that $g \circ \gamma \in\left\langle\phi \circ \gamma, B_{1} \circ \gamma, \ldots, B_{r} \circ \gamma\right\rangle$. Then, for all $\gamma: \mathbb{C} \rightarrow \mathbb{C} \times X \subset \mathbb{C} \times \mathbb{C}^{n}$ we have that $g \circ \gamma \in\left\langle B_{1} \circ \gamma, \ldots, B_{r} \circ \gamma\right\rangle$. Again by Theorem 2.1 we concluded that $g \in \overline{J_{X}}$ in $\mathcal{O}_{\mathbb{C} \times X}$.

With this lemma, in the following corollaries, we relate the Newton polyhedron to the item $\left(4_{X}\right)$ of our Theorem 3.4 .

Corollary 4.3. If $\Gamma_{+}(g) \subset C(\overline{\langle\phi\rangle+J(f, \phi)})$ in $\mathcal{O}_{n+1}$ and $\Gamma_{+}\left(C_{i}\right) \subset C(\overline{\langle\phi\rangle+J(f, \phi)})$ in $\mathcal{O}_{n+1}$ for $i=1, \ldots, r$ then $g \in \overline{J_{X}}$ in $\mathcal{O}_{\mathbb{C} \times X}$.

Proof: Since $\Gamma_{+}(g) \subset C(\overline{\langle\phi\rangle+J(f, \phi)})$ in $\mathcal{O}_{n+1}$ and $\Gamma_{+}\left(C_{i}\right) \subset C(\overline{\langle\phi\rangle+J(f, \phi)})$ in $\mathcal{O}_{n+1}$ then $g \in \overline{\langle\phi\rangle+J(f, \phi)}$ in $\mathcal{O}_{n+1}$ and $C_{i} \in \overline{\langle\phi\rangle+J(f, \phi)}$ in $\mathcal{O}_{n+1}$.

Thus, by the Lemma 4.2, $g \in \bar{J}_{X}$ in $\mathcal{O}_{\mathbb{C} \times X}$.

Corollary 4.4. If $\langle\phi\rangle+J(f, \phi)$ is Newton non-degenerate, $\Gamma_{+}(g) \subset \Gamma_{+}(\langle\phi\rangle+J(f, \phi))$ in $\mathcal{O}_{n+1}$ and $\Gamma_{+}\left(C_{i}\right) \subset \Gamma_{+}(\langle\phi\rangle+J(f, \phi))$ in $\mathcal{O}_{n+1}$ for $i=1, \ldots, r$ then $g \in \overline{J_{X}}$ in $\mathcal{O}_{\mathbb{C} \times X}$.

Proof: Since $\langle\phi\rangle+J(f, \phi)$ is Newton non-degenerate then by Theorem 4.1 we have that $\Gamma_{+}(\langle\phi\rangle+J(f, \phi))=C(\overline{\langle\phi\rangle+J(f, \phi)})$.

Therefore, by the Corollary 4.3, $g \in \bar{J}_{X}$ in $\mathcal{O}_{\mathbb{C} \times X}$.

We are ready, now, to produce interesting examples of families with constant Milnor number. We highlight that the families in the two following examples do not satisfy the hypothesis of the Theorem 3.5.

Example 4.5. Let $(X, 0) \subset\left(\mathbb{C}^{3}, 0\right)$ be defined by $\phi(x, y, z)=\left(x y, x^{15}+y^{10}+z^{6}\right)$ and let $f:(X, 0) \rightarrow(\mathbb{C}, 0)$ be defined by $f(x, y, z)=x+z$. We have that $\phi$ and $f$ are weighted homogeneous with different weights. Therefore, we can not use the Theorem 3.5. Let $F:(\mathbb{C} \times X) \rightarrow(\mathbb{C}, 0)$ be the deformation of $f$ defined by $F(t,(x, y, z))=x+z+t x y$.

We have that $\langle\phi\rangle+J(f, \phi)=\left\langle x y, x^{15}+y^{10}+z^{6}, 6 x z^{5}+10 y^{10}-15 x^{15}\right\rangle$ is Newton non-degenerate, $\Gamma_{+}(g) \subset \Gamma_{+}(\langle\phi\rangle+J(f, \phi))$ in $\mathcal{O}_{n+1}$ and $\Gamma_{+}\left(C_{1}\right) \subset \Gamma_{+}(\langle\phi\rangle+J(f, \phi))$ in $\mathcal{O}_{n+1}$, where $g(x, y, z)=x y$ and $C_{1}$ is the determinant of $J(g, \phi)$. Then, by Corollary 4.4, $\partial F / \partial t \in \overline{J_{X}}$ in $\mathcal{O}_{\mathbb{C} \times X}$. Therefore, by Theorem [3.4, $F$ is $\mu$-constant.

Example 4.6. Let $(X, 0) \subset\left(\mathbb{C}^{3}, 0\right)$ be defined by $\phi(x, y, z)=x^{3}+y^{3}+z^{4}+x y z$ and let $f:(X, 0) \rightarrow(\mathbb{C}, 0)$ be defined by $f(x, y, z)=x y+z^{2}$. Consider $F:(\mathbb{C} \times X) \rightarrow(\mathbb{C}, 0)$ be the deformation of $f$ defined by $F(t,(x, y, z))=f(x, y, z)+\operatorname{tg}(x, y, z)$. Note that $\phi$ is not weighted homogeneous.

We have that $\partial F / \partial t=g$ and $\langle\phi\rangle+J(f, \phi)=\left\langle x^{3}+y^{3}+z^{4}+x y z,-x^{2} y+6 y^{2} z+2 x z^{2}-\right.$ $\left.4 x z^{3},-x y^{2}+6 x^{2} z+2 y z^{2}-4 y z^{3},-3 x^{3}+3 y^{3}\right\rangle$ is Newton non-degenerate. In addition, except for $g=z^{3}$ any other deformation with a degree greater or equal to three is such that $\Gamma_{+}(g) \subset \Gamma_{+}(\langle\phi\rangle+J(f, \phi))$ in $\mathcal{O}_{n+1}$ and $\Gamma_{+}\left(C_{i}\right) \subset \Gamma_{+}(\langle\phi\rangle+J(f, \phi))$ in $\mathcal{O}_{n+1}$, where $C_{i}$ are the minors of order 2 of $J(g, \phi)$ for $i=1,2,3$. Thus, by Corollary 4.4, $\partial F / \partial t \in \overline{J_{X}}$ in $\mathcal{O}_{\mathbb{C} \times X}$. Therefore, $F$ is $\mu$-constant.

\section{Other INVARIANTS}

Given an ICIS $(X, 0) \subset\left(\mathbb{C}^{n}, 0\right)$, we have two important subgroups of the $\mathcal{R}$ group of diffeomorphisms from $\left(\mathbb{C}^{n}, 0\right)$ to $\left(\mathbb{C}^{n}, 0\right)$ : one is the group $\mathcal{R}_{X}$ of the diffeomorphisms which preserves $(X, 0)$, the other is $\mathcal{R}(X)$, the group of diffeomorphisms of $X$. We know 
that if the germs $f, g:(X, 0) \rightarrow(\mathbb{C}, 0)$ are $\mathcal{R}_{X}$-equivalent then they are $\mathcal{R}(X)$-equivalent, but the converse is not true.

In the smooth case, we know that a germ $f:\left(\mathbb{C}^{n}, 0\right) \rightarrow(\mathbb{C}, 0)$ is finitely determined if and only if $\mu(f)$ is finite. There exist a generalization of this result for the $\mathcal{R}_{X}$-group: $f:\left(\mathbb{C}^{n}, 0\right) \rightarrow(\mathbb{C}, 0)$ is $\mathcal{R}_{X}$-finitely determined if and only if $\mu_{B R}(X, f)$ is finite. Here $\mu_{B R}(X, f)$ is the Bruce-Roberts number defined in [3] in the following by

$$
\mu_{B R}(X, f)=\operatorname{dim}_{\mathbb{C}} \frac{\mathcal{O}_{n}}{J_{f}\left(\Theta_{X}\right)},
$$

where $\Theta_{X}$ is the $\mathcal{O}_{n}$-module of vector fields in $\left(\mathbb{C}^{n}, 0\right)$ which are tangent to $(X, 0)$ and $J_{f}\left(\Theta_{X}\right)=\left\langle d f(\xi) \mid \xi \in \Theta_{X}\right\rangle$.

Because of this, it is important to know when a family has constant Bruce-Roberts number.

Another important number related to $f:(X, 0) \rightarrow(\mathbb{C}, 0)$ is the Euler obstruction, $E u_{f, X}(0)$. This number is very studied for instance in [2] and [9].

In this section we use our results to conclude when the Bruce-Roberts number or the Euler obstruction of a family $f_{t}:(X, 0) \rightarrow(\mathbb{C}, 0)$ is constant. Also, we present a sufficient condition for such a family to be $C^{0}-\mathcal{R}_{X}\left(\right.$ or $\left.C^{0}-\mathcal{R}(X)\right)$-trivial.

In [1, Ahmed, Ruas and Tomazella studied the constancy of the Bruce-Roberts number of a family. In order to understand this, we need to know the logarithmic characteristic variety.

Let $U \subset \mathbb{C}^{n}$ be a neighborhood of origin. Suppose that $\Theta_{X}=\left\langle\xi_{1}, \ldots, \xi_{p}\right\rangle$ em $U$. We define

$$
L C_{U}(X):=\left\{(x, \delta) \in T_{U}^{*} \mathbb{C}^{n} \mid \delta\left(\xi_{i}(x)\right)=0, i=1, \ldots, p\right\},
$$

where $T_{U}^{*} \mathbb{C}^{n}$ is the restriction of the cotangent bundle of $\mathbb{C}^{n}$ to $U$.

The logarithmic characteristic variety of $X$, which we denote by $L C(X)$, is defined as the germ of $L C_{U}(X)$ in $T_{0}^{*} \mathbb{C}^{n}$, the cotangent space of $\mathbb{C}^{n}$ in 0 .

Theorem 5.1. [1, Theorem 3.11] Suppose that $X$ is a hypersurface with isolated singularity and $L C(X)$ is Cohen-Macaulay, $f:\left(\mathbb{C}^{n}, 0\right) \rightarrow(\mathbb{C}, 0)$ a germ with isolated singularity and $F:\left(\mathbb{C} \times \mathbb{C}^{n}, 0\right) \rightarrow(\mathbb{C}, 0)$ a deformation of $f$. If $\partial F / \partial t \in \overline{J_{F}\left(\Theta_{X}\right)}$ then $F$ is a $\mu_{B R}$-constant deformation of $f$.

In addition, given $(X, 0) \subset\left(\mathbb{C}^{n}, 0\right)$ an ICIS, Grulha in [9] proved the equivalence between the constancy of $\mu\left(X \cap f_{t}^{-1}(0), 0\right)$ and $E u_{f, X}(0)$, by the Lê-Greuel formula this show the importance of our Theorem 3.4 .

Theorem 5.2. [9, Proposition 5.17] Let $(X, 0) \subset\left(\mathbb{C}^{n}, 0\right)$ be an ICIS, and $F:\left(\mathbb{C}^{n} \times\right.$ $\left.\mathbb{C}^{r}, 0\right) \rightarrow \mathbb{C}$ a family of functions with isolated singularity, where $F(t, x)=f_{t}(x)$. Then, the following statements are equivalent:

(1) $E u_{f_{t}, X}(0)$ is constant for the family.

(2) $\mu\left(X \cap f_{t}^{-1}(0), 0\right)$ is constant for the family.

Using these results and the Theorem 3.4 we obtain the following applications of our study:

Theorem 5.3. Let $(X, 0) \subset\left(\mathbb{C}^{n}, 0\right)$ be an ICIS, $f:(X, 0) \rightarrow(\mathbb{C}, 0)$ a germ with isolated singularity $\mathcal{R}_{X}$-finitely determined and $F:(\mathbb{C} \times X, 0) \rightarrow(\mathbb{C}, 0)$ a deformation of $f$. If $\partial F / \partial t \in \overline{J_{X}}$ then:

(i) $\tilde{F}$ is $C^{0}-\mathcal{R}_{X}$-trivial (and therefore $\tilde{F}$ is $C^{0}-\mathcal{R}(X)$-trivial), where $\tilde{F}:\left(\mathbb{C} \times \mathbb{C}^{n}, 0\right) \rightarrow$ $(\mathbb{C}, 0)$ is such that $F=\left.\tilde{F}\right|_{X}$. 
(ii) $E u_{\tilde{f}_{t}, X}(0)$ is constant, where $\tilde{f}_{t}:\left(\mathbb{C}^{n}, 0\right) \rightarrow(\mathbb{C}, 0)$ is such that $f_{t}=\left.\tilde{f}_{t}\right|_{X}$.

(iii) If $(X, 0)$ is a hypersurface with isolated singularity and $L C(X)$ Cohen-Macaulay then $\mu_{B R}\left(X, \tilde{f}_{t}\right)$ is constant.

(iv) If $(X, 0)$ is a weighted homogeneous hypersurface with isolated singularity then $\mu_{B R}\left(X, \tilde{f}_{t}\right)$ is constant and $m\left(\tilde{f}_{t}\right)$ is constant, where $m\left(\tilde{f}_{t}\right)$ is the multiplicity of $\tilde{f}_{t}$.

\section{Proof:}

(i) In [21, Theorem 4.3] it is shown that if $(X, 0)$ is an ICIS and $\partial F / \partial t \in \overline{J_{F}\left(\Theta_{X}\right)}$ then $\tilde{F}$ is $C^{0}-\mathcal{R}_{X^{-}}$trivial. Therefore, as $J_{X} \subseteq J_{F}\left(\Theta_{X}\right)$ we have the desired. Thus, $\tilde{F}$ also is $C^{0}-\mathcal{R}(X)$-trivial.

(ii) Follows directly from Theorem 3.4 and Theorem 5.2 .

(iii) Just use $J_{X} \subseteq J_{F}\left(\Theta_{X}\right)$ and Theorem 5.1 .

(iv) In [19, Theorem 4.2] it is shown that if $(X, 0)$ is a weighted homogeneous hypersurface with isolated singularity, then $L C(X)$ is Cohen-Macaulay. Then follows as a consequence of item (iii) that $\mu_{B R}\left(X, \tilde{f}_{t}\right)$ is constant. Thus, $m\left(\tilde{f}_{t}\right)$ is constant (see [1, Theorem 4.3]).

As an application of the item (ii) of the Theorem 5.3 we have that the families of Examples 4.5 and 4.6 have constant Euler obstruction.

\section{Deformation of The ICIS}

Our final goal is to answer a natural question: What if we deform the ICIS instead of the function germ?

Let $\phi:\left(\mathbb{C}^{n}, 0\right) \rightarrow\left(\mathbb{C}^{p}, 0\right)$ be a holomorphic map germ and let $(X, 0) \subset\left(\mathbb{C}^{n}, 0\right)$ be the ICIS defined by the zero set of $\phi$.

Let $\Phi:\left(\mathbb{C} \times \mathbb{C}^{n}, 0\right) \rightarrow\left(\mathbb{C}^{p}, 0\right)$ be a deformation of $\phi$, defined by $\Phi(t, x)=\phi_{t}(x)$, such that $\phi_{0}=\phi$ and $\left(X_{t}, 0\right):=\left(\phi_{t}^{-1}(0), 0\right)$ is an ICIS for $t$ small enough. We denote $\mathcal{X}=\Phi^{-1}(0)$. Let $f:\left(\mathbb{C}^{n}, 0\right) \rightarrow(\mathbb{C}, 0)$ be a holomorphic function germ such that

$$
\begin{aligned}
f_{t}:\left(X_{t}, 0\right) & \rightarrow(\mathbb{C}, 0) \\
x & \mapsto f(x)
\end{aligned}
$$

has isolated singularity for $t$ small enough. We study here the constancy of the Milnor number $\mu\left(\left.f_{t}\right|_{X_{t}}\right)$.

Consider $G: \mathbb{C} \times \mathbb{C}^{n} \rightarrow \mathbb{C} \times \mathbb{C}^{p}$ defined by $G(t, x)=\left(f(x), \phi_{t}(x)\right)$.

For the case where we did not deform $X$, we saw, in the Example 3.3, that $\partial G / \partial t \in$ $\overline{\left\{\partial G / \partial x_{j}\right\}}$ in $\mathcal{O}_{X \cap f_{t}^{-1}(0)}$ does not imply that $f_{t}$ is $\mu$-constant. We know that if $\partial G / \partial t \in$ $\overline{\left\{x_{i}\left(\partial G / \partial x_{j}\right)\right\}}$ in $\mathcal{O}_{X_{t} \cap f_{t}^{-1}(0)}$ then $\left(\mathcal{X}_{0}, \mathbb{C} \times\{0\}\right)$ is Whitney regular (see [5, Theorem 2.5]), where $\mathcal{X}_{0}=\mathcal{X}-\mathbb{C} \times\{0\}$. We also know that if $\left.\partial G / \partial t \in \overline{\left\{\partial G / \partial x_{j}\right\}}\right\}^{\dagger}$ then $A_{f_{t}}$ holds for the pair $\left(\mathcal{X}_{0}, \mathbb{C} \times\{0\}\right)$ (see [7, Lemma 5.1]) (see definition of $A_{f_{t}}$ in [8]). Hence, we can think about the relationship between $\partial G / \partial t \in{\overline{\left\{\partial G / \partial x_{j}\right\}}}^{\dagger}$ and the constancy of $\mu\left(f_{t}\right)$.

Theorem 6.1. The following statements are equivalent:

$\left(1_{X t}\right) F$ is $\mu$-constant.

$\left(\mathscr{Q}_{X t}\right) \partial G / \partial t \in \overline{\left\{\partial G / \partial x_{j}\right\}}{ }^{\dagger}$.

$\left(3_{X t}\right) v\left(J_{X}\right)=\left\{(t, x) \in \mathbb{C} \times \mathbb{C}^{n} \mid B_{i}(t, x)=0, i=1, \ldots, r\right\}=\mathbb{C} \times\{0\}$ near $(0,0)$, where $B_{1}, \ldots, B_{r}$ are the maximal order minors of the Jacobian matrix of $\left(f, \phi_{t}\right)$ (partial derivatives with respect to $x$, only) and $J_{X}$ is the ideal generated by them. 


\section{Proof:}

$\left(1_{X_{t}}\right) \Leftrightarrow\left(3_{X_{t}}\right)$ :

It is the proof of $\left(1_{X}\right) \Leftrightarrow\left(6_{X}\right)$ in Theorem 3.4 .

$\left(1_{X_{t}}\right) \Rightarrow\left(2_{X_{t}}\right)$ :

Suppose $F$ is $\mu$-constant. By the Lê-Greuel formula, we have that $\mu\left(\left.f_{t}\right|_{X}\right)=\mu\left(X_{t}, 0\right)+$ $\mu\left(X_{t} \cap f_{t}^{-1}(0), 0\right)$. Thus, $\mu\left(X_{t}, 0\right)$ and $\mu\left(X_{t} \cap f_{t}^{-1}(0), 0\right)$ are constants. Then, $A_{f_{t}}$ holds for the pair $\left(\mathcal{X}_{0}, \mathbb{C} \times\{0\}\right)$ (see [8, Theorem 5.8]). Therefore, $\partial G / \partial t \in \overline{\left\{\partial G / \partial x_{j}\right\}}{ }^{\dagger}$ (see [7, Lemma 5.1]).

$$
\left(2_{X_{t}}\right) \Rightarrow\left(1_{X_{t}}\right) \text { : }
$$

Suppose that $\partial G / \partial t \in{\overline{\left\{\partial G / \partial x_{j}\right\}}}^{\dagger}$ then $A_{f_{t}}$ holds for the pair $\left(\mathcal{X}_{0}, \mathbb{C} \times\{0\}\right)$ (see [7, Lemma 5.1]). Hence, the Buchsbaum-Rim multiplicity of the module $\left\{\partial G / \partial x_{j}\right\}$ is constant (see [12, Theorem 3.2]). Thus, $F$ is $\mu$-constant (see [12, Lemma 3.3]).

\section{Acknowledgements}

We thank A. Miranda and T. Gaffney for the fruitful discussion on the issue.

\section{REFERENCES}

1. I. Ahmed, M. A. S. Ruas and J. N. Tomazella, Invariants of topological relative right equivalences, Math. Proc. Cambridge Philos Soc. 155 (2013), No. 2, 307-315.

2. J.-P. Brasselet, D. Massey, A. J. Parameswaran and J. Seade, Euler obstruction and defects of functions on singular varieties, J. London Math. Soc. (2) 70 (2004), No. 1, 59-76.

3. J. W. Bruce and R. M. Roberts, Critical points of functions on analytic varieties, Topology 27, (1988), No. 1, 57-90.

4. T. Gaffney, Equisinularity of plane sections, $t_{1}$ condition and the integral closure of modules, Real and complex singularities (São Carlos, 1994), Pitman Res. Notes Math. Ser. 333, Longman, Harlow, (1995), 95-111.

5. T. Gaffney, Integral closure of modules and Whitney equisingularity, Invent. Math. 107 (1992), No. $2,301-322$.

6. T. Gaffney, Polar multiplicities and equisingularity of map germs, Topology 32 (1993), No.1, 185-223.

7. T. Gaffney and S. L. Kleiman, Specialization of integral dependence for modules, Invent. Math. 137 (1999), No. 3, 541-574.

8. T. Gaffney and D. Massey, Trends in equisingularity theory, Singularity theory (Liverpool, 1996), xix-xx, London Math. Soc. Lecture Notes Ser. 263, Cambridge Univ. Press, Cambridge, (1999), 207-248.

9. N. de Góes Grulha Jr., The Euler obstruction and Bruce-Roberts' Milnor number, Q. J. Math. 60 (2009), No. 3, 291-302.

10. G. M. Greuel, Constant Milnor number implies constant multiplicity for quasihomogeneous singularities, Manuscripta Math. 56 (1986), No. 2, 159-166.

11. H. Hamm, Lokale topologische Eigenschaften Komplexer Räume, Math. Ann. 191 (1971), 235-252.

12. S. L. Kleiman, Equisingularity, Multiplicity, and Dependence, Commutative algebra and algebraic geometry (Ferrara), Lecture Notes in Pure and Appl. Math., 206 Dekker, New York, (1999), 211225 .

13. Dũng Tráng, Lê and C. P. Ramanujam, The invariance of Milnor's number implies the invariance of topological type, Amer. J. Math. 98 (1976) No. 1, 67-78.

14. E. J. N. Looijenga, Isolated singular points on complete intersections, London Mathematical Society Lecture Note Series, 77. Cambridge University Press (1984).

15. H. Matsumura, Commutative ring theory, Cambridge University Press, (1989).

16. J. Milnor, Singular points of complex hypersurfaces, Annals of Math. Studies, Princeton University Press (1968). 
17. D. Mumford, Algebraic Geometry. I. Complex projective varieties, In A series of comprehensive studies in Mathematics, vol. 221 Springer, (1976).

18. J. J. Nuño-Ballesteros, B. Oréfice-Okamoto and J. N. Tomazella, Non-negative deformations of weighted homogeneous singularities, Glasg. Math. J. 60 (2018), No. 1, 175-185.

19. J. J. Nuño-Ballesteros, B. Oréfice and J. N. Tomazella, The Bruce-Roberts number of a function on a weighted homogeneous hypersurface. Q. J. Math. 64 (2013), No. 1, 269-280.

20. A. J. Parameswaran, Topological equisingularity for isolated complete intersection singularities, Compositio Math. 80 (1991), No. 3, 323-336.

21. M. A. S. Ruas and J. N. Tomazella, An infinitesimal criterion for topological triviality of families of sections of analytic varieties, Singularity theory and its applications, Adv. Stud. Pure Math. 43 (2006), 421-436.

22. M. J. Saia, The integral closure of ideals and the Newton filtration, J. Algebraic Geom. 5 (1996), No. $1,1-11$.

23. B. Teissier, Cycles évanescents: sections planes et conditions de Whitney, (French) Singularités à Cargèse, (Rencontre Singularités Géom. Anal., Inst. Études Sci., Cargèse, 1972) Astérisque, Nos. 7 et 8, Soc. Math. France, Paris (1973), 285-362.

24. J. G. Timourian, Invariance of Milnor's number implies topological triviality, Amer. J. Math. 99 (1977), No. 2, 437-446.

Departamento de Matemática, Universidade Federal de São Carlos, Caixa Postal 676, 13560-905, SÃo CARLOS, SP, BRAZIL

E-mail address: rafaelasoares@dm.ufscar.br

Departamento de Matemática, Universidade Federal de São Carlos, Caixa Postal 676, 13560-905, SÃo CARLOS, SP, BRAZIL

E-mail address: bruna@dm.ufscar.br

Departamento de Matemática, Universidade Federal de São Carlos, Caixa Postal 676, 13560-905, SÃo CARlos, SP, BRAZIL

E-mail address: tomazella@dm.ufscar.br 